\title{
The Increase in Prevalence and Incidence of Depression in Korea from 2002 to 2013
}

\section{Dongjun Shin ${ }^{a}$, Ga Eun Kim ${ }^{b}$, Seung Woo Cho ${ }^{c}$, Min-Woo Jo ${ }^{d^{*}}$, Yong-Wook Shin ${ }^{a^{*}}$}

a Department of Psychiatry, University of Ulsan College of Medicine, Asan Medical Center, Seoul, South Korea

${ }^{b}$ Department of Psychiatry, College of Medicine, Ewha Womans University, Seoul, South Korea

'Department of Occupational and Environmental Medicine, Ulsan University Hospital, University of Ulsan College of Medicine, Ulsan, South Korea

${ }^{d}$ Department of Preventive Medicine, University of Ulsan College of Medicine, Seoul, South Korea

* Corresponding Authors

\section{INTRODUCTION}

- South Korea has the highest suicide rate among OECD countries, about 2.4 times higher than the average suicide rate of other OECD countries.

- Despite the high suicide rate, the prevalence of depression in Korea has been reported to be much lower than in other countries.

- In the previous cross-sectional national surveys, the study populations were convenient and different in each study, and the number of subjects in the target population was small.

- We analyzed the National Health Insurance Service-National Sample Cohort (NHIS-NSC) to estimate the prevalence and incidence of the depressive disorder and changes in the Korean population over the decade.

\section{METHODS}

\section{NHIS-NSC Database}

- We analyzed the NHIS-NSC database (2002-2013) which has been implemented by the NHIS, a single national insurance provider in Korea.

- The cohort consists of a nationally representative sample having the clinical record of about one million participants randomly selected from approximately $97 \%$ of the overall population of Korea from 2002 to 2013.

Operational definition of depression

- The prevalence and incidence of depression was identified when the patient had a claim record with a diagnosis of depression based on the ICD-10, or the ICD-10 and a record of prescription of antidepressants.

- We calculated each annual age- or sex-standardized prevalence of depressive disorder.

\section{RESULTS}

- In 2002, among total 1,025,340 participants of NHIS-NCS database, the number of men and women was each 513,258 and 512,082.

- Among the participants in $2002,4,380(0.85 \%)$ male participants were diagnosed to have a depressive disorder, and 1,276 (0.25\%) had a diagnosis of depressive disorder and the prescription of antidepressants. Among female participants, 8,935 (1.74\%) participants were diagnosed to have a depressive disorder, and $2,834(0.55 \%)$ had both the diagnosis and the prescription.

- In 2013 , a total of $1,014,730$ participants were enrolled in the database.

- Among 507,289 male participants in $2013,13,809$ (2.72\%) participants were diagnosed to have a depressive disorder, and $6,863(1.35 \%)$ had both the diagnosis and the prescription. Among 507,441 female participants in 2013, 24,171 (4.76\%) participants were diagnosed with depressive disorder, and 12,472 (2.46\%) had both.
Figure1. Prevalence of depressive disorder from 2002 to 2013.

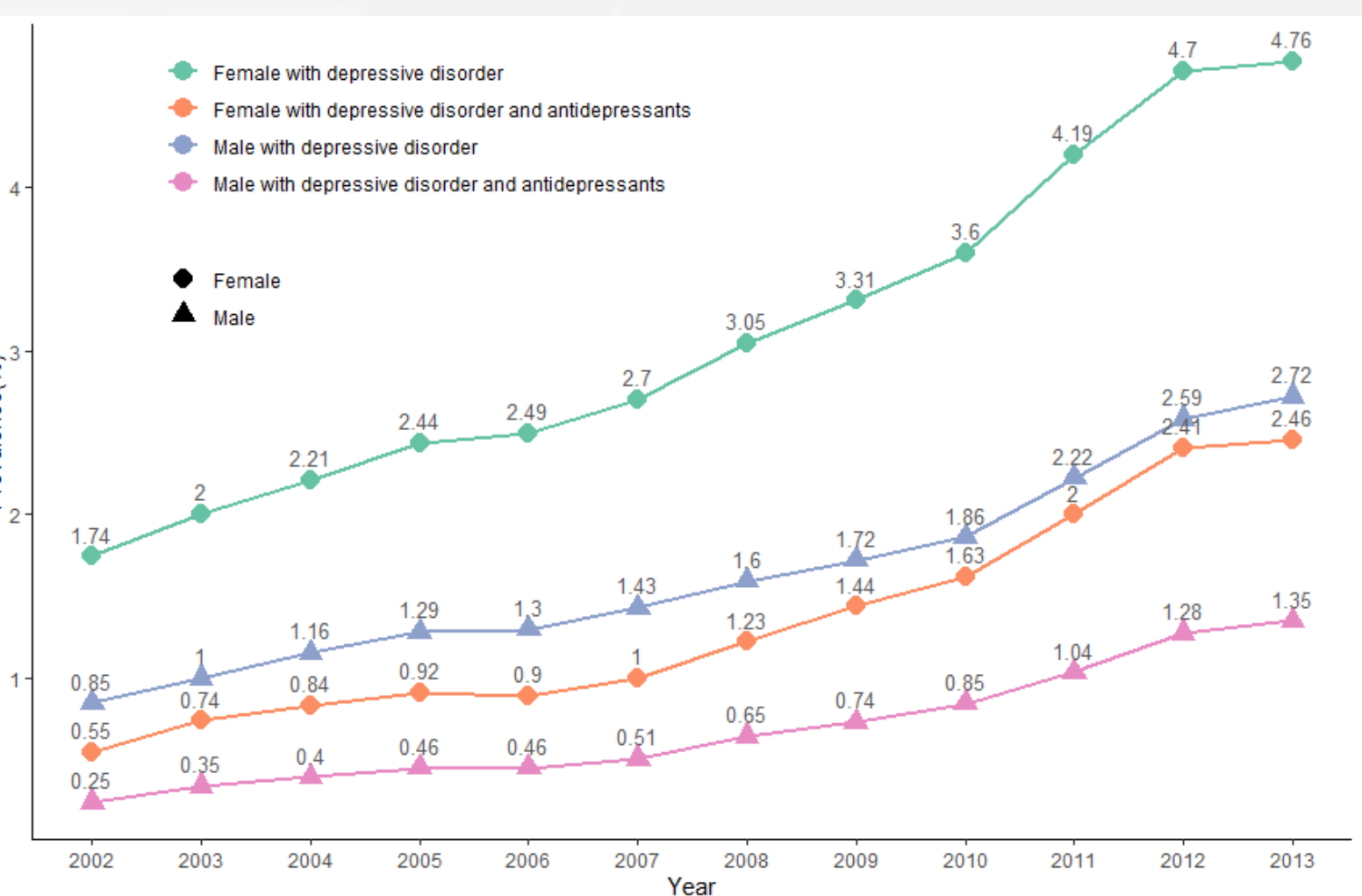

Figure2. Prevalence of depressive disorder in 2002 and 2013 according to the age group.

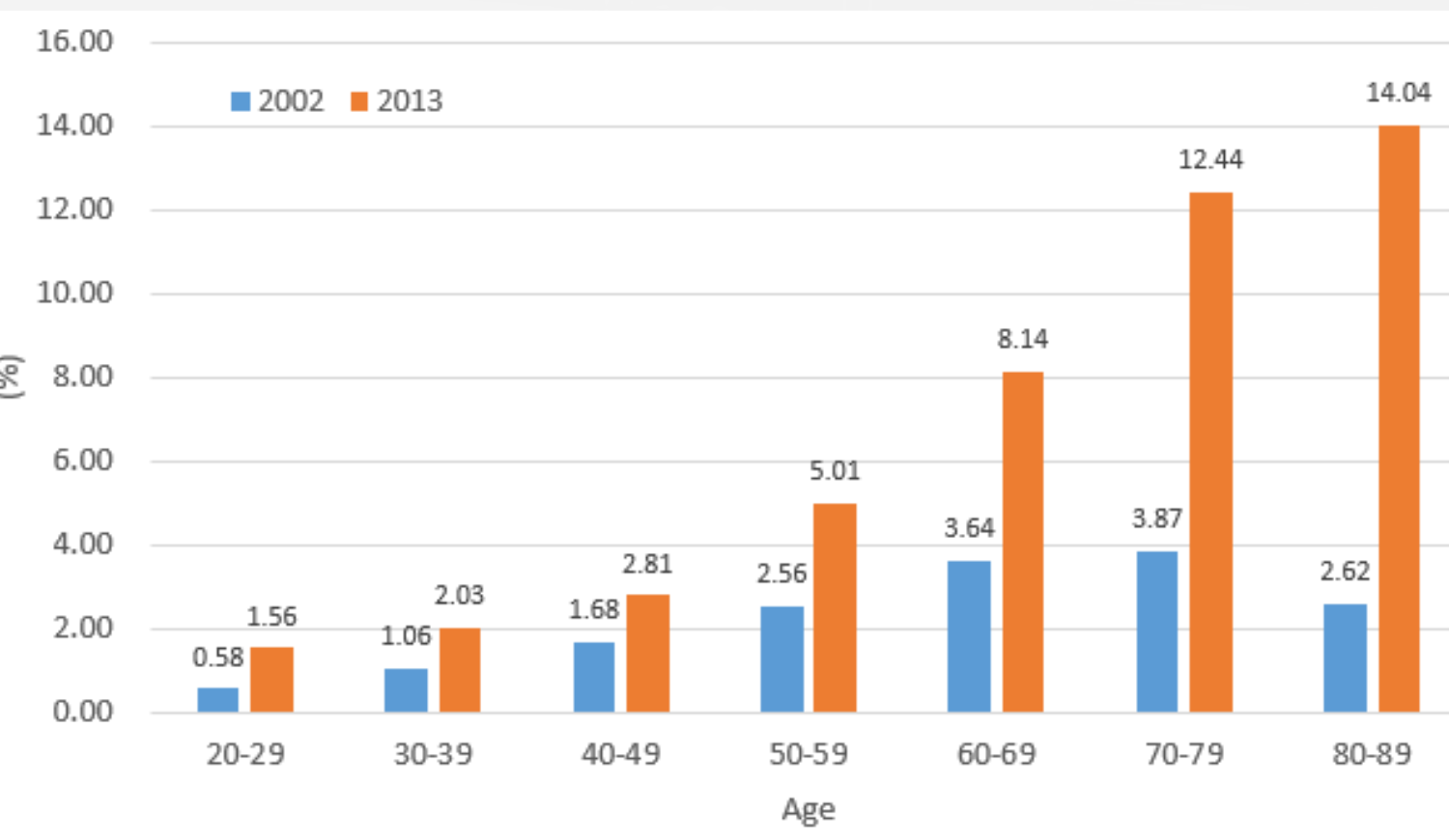

- The prevalence of depression in the total population in 2013 were $3.81 \%$. In females, the prevalence was $2.46 \%$ which was higher than in males with $1.35 \%$.

- During the period from 2002 to 2013, the standardized prevalence rate of depression increased from $0.80 \%$ in 2002 to $3.81 \%$ in 2013.

- As the age of the population increased, the prevalence of depressive disorder increased.

- The prevalence of depression in those in their 80s and above was about seven times that of those in their 20 s.

\section{CONCLUSION}

- The increase in the prevalence of depression was rapid from 2002 to 2013. The prevalence of depression showed the highest increase rate among the elderly in the 80s and above.

- This national sample cohort study with the large representative sample data provided convincing evidence of the increased prevalence of depression in South Korea during the recent decade. 